\title{
Learning Phase Retrieval with Backpropagation
}

Youssef S. G. Nashed ${ }^{1 *}$, Saugat Kandel ${ }^{2}$, Ming Du ${ }^{3}$ and Chris Jacobsen ${ }^{4,5}$

1. Mathematics and Computer Science Division, Argonne National Laboratory, Lemont, IL 60439, USA.

2. Applied Physics, Northwestern University, Evanston, IL 60208, USA.

3. Department of Materials Science and Engineering, Northwestern University, Evanston, IL 60208, USA.

4. Department of Physics \& Astronomy, Northwestern University, Evanston, IL 60208, USA.

5. Advanced Photon Source, Argonne National Laboratory, Lemont, IL 60439, USA.

* Corresponding author: ynashed@anl.gov

Synchrotron radiation light source facilities are leading the way to ultrahigh resolution X-ray imaging. High resolution imaging is essential to understanding the fundamental structure and interaction of materials at the smallest length scale possible. Coherent diffraction imaging (CDI) achieves nanoscale imaging by replacing traditional objective lenses by pixelated area detectors and computational image reconstruction. We present our work for solving CDI reconstruction problems through fitting a physics based model to measured data. The model parameters are learned in a similar manner to deep neural networks, utilizing the backpropagation method as implemented in Google TensorFlow package. This approach has advantages in terms of speed and accuracy compared to current state of the art algorithms, and demonstrates re-purposing the deep learning backpropagation algorithm to solve general phase retrieval problems that are prevalent in lensless microscopy research.

Solving the challenging phase retrieval problem, where one attempts to reconstruct an object from only the magnitude of its Fourier transform, requires the use of oversampling and support constraints. These are typically used in an iterative projection framework that updates the object guess by applying a Fourier magnitude projection and a real-space constraint projection [1]. Alternatively, we can also frame phase retrieval as a nonlinear minimization problem, where we minimize an error metric using a gradient-based approach. The gradient-based approach is flexible and can include in the forward model

a large variety of the physical phenomena related to the probing light (such as partial coherence, source fluctuations, and experimental errors), or the detection process (such as the measurement noise and the finite size of the pixel).

Gradient-based phase retrieval methods in the literature tend to rely on the availability of a closed-form expression for the gradient calculation. This closed-form expression is typically obtained by writing down an explicit expression for the error metric to minimize, then symbolically differentiating the error metric with respect to the individual input parameters [2]. Calculating the gradient in this fashion is laborious; a slight modification of the forward model usually requires a complete rederivation and algorithmic reimplementation of the gradient expressions. This becomes especially limiting if we desire to explore variations of, or introduce new capabilities to, our basic experimental methodology. As such, it is more than desirable to have an approach beyond symbolic differentiation in order to easily explore a variety of algorithms and approaches.

Automatic differentiation or Algorithmic Differentiation (AD), provides such an alternative to symbolic differentiation. This approach is based upon the observation that vector-valued functions can, in general, be interpreted as composites of basic arithmetic operations and a limited number of elementary functions 
(including exponentials and trigonometric operations). Differentiation of functions can then be understood as a recursive application of the chain rule of differentiation, wherein we repeatedly differentiate the same elementary functions (with known derivatives), only with different input parameters. Here we use the backpropagation algorithm used to train deep neural networks is a special case of reverse mode AD [3]. Consequently, several software libraries available for deep learning also include high performance routines for $\mathrm{AD}$. TensorFlow is a Python based deep learning API provided by Google. Here we use TensorFlow to automatically calculate gradients for different phase retrieval forward models, in order to highlight the flexibility of the approach. The physics-based forward model is the only part that needs to be explicitly specified. The solution to the associated inverse phase retrieval problem is achieved through the repeated application of the backpropagation algorithm to an initial random guess. In previous publications we demonstrate f our approach for various ptychography forward models, namely: far-field ptychography [4], near-field ptychography, and multi-angle Bragg ptychography [5].

The use of $\mathrm{AD}$ not only provides an alternative to current 2D CDI reconstruction methods but also allow one to easily extend to $3 \mathrm{D}$ reconstruction with more complicated physics models. Recently, by modelling wave propagating in the sample volume with the multislice method, we have developed a novel reconstruction algorithm that addresses the multiple scattering of x-ray in thick objects, which enables the retrieval of samples beyond the depth of focus. Shown in Fig. 1 is the output of a thick object using the described method, where the reconstruction quality is much better than that obtained using conventional methods (error reduction + filtered back projection).

\section{References:}

[1] JR Fienup, Applied Optics 21 (1982), p. 2758-2769.

[2] AS Jurling and JR Fienup, JOSA A 31.7 (2014), p. 1348-1359.

[3] K Schmidhuber, Neural Networks 61 (2015), p.85-117.

[4] YSG Nashed et al., Procedia Computer Science 108 (2017), p.404-414.

[5] S Kandel et al., arXiv preprint arXiv:1902.03920 (2019) .

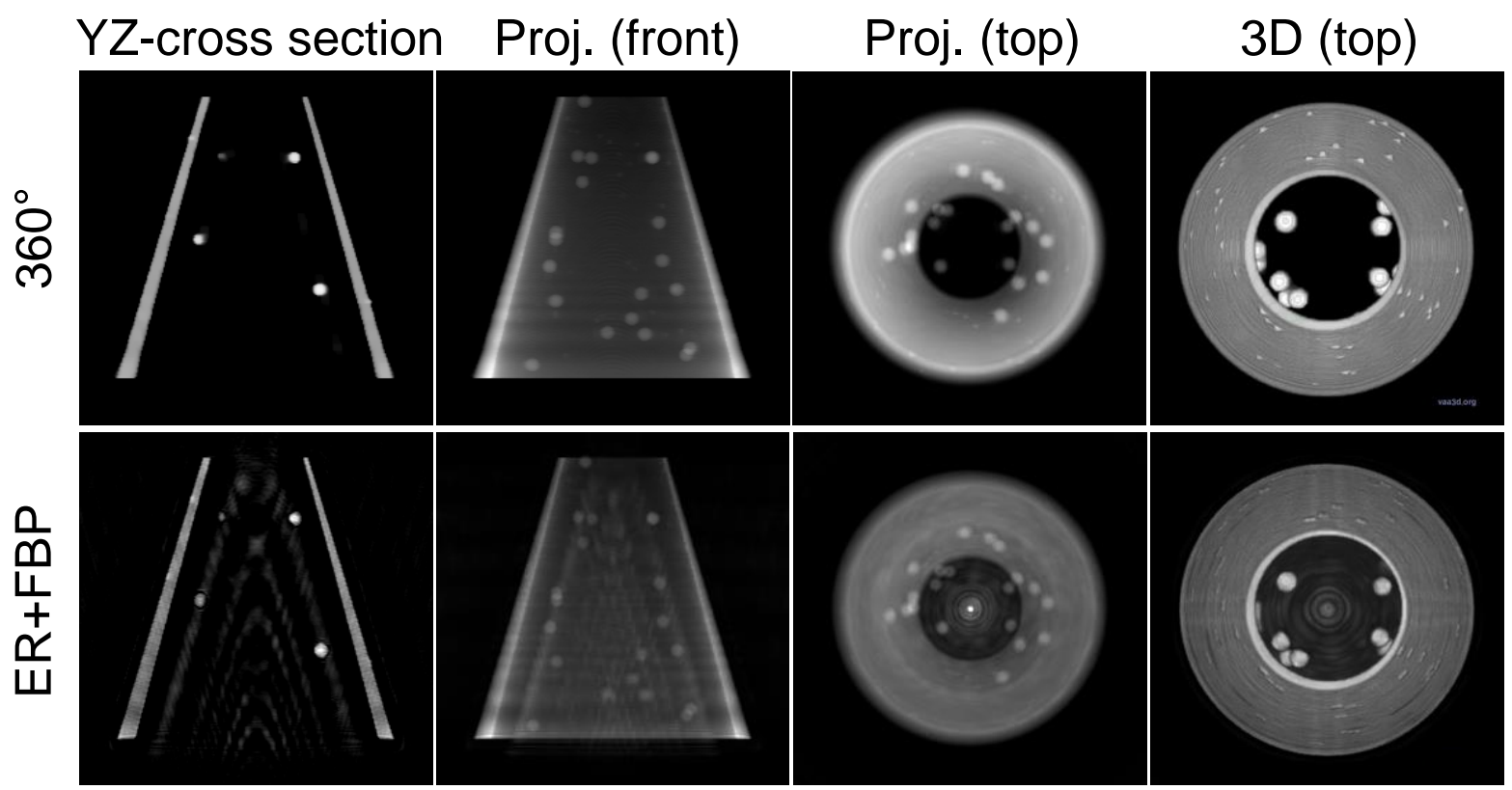

Figure 1. Comparison between our approach (top) and the standard method (bottom), for 3D beyond depth-of-focus ptychographic reconstruction of a simulated sample. 